\title{
Magnetoelastic coupling enabled tunability of magnon spin current generation in 2D antiferromagnets
}

\author{
N. Bazazzadeh, ${ }^{1}$ M. Hamdi, ${ }^{1, *}$ S. Park,${ }^{2,3,4}$ A. Khavasi,${ }^{5}$ S. M. Mohseni, ${ }^{1, \dagger}$ and A. Sadeghi ${ }^{1, \ddagger}$ \\ ${ }^{1}$ Department of Physics, Shahid Beheshti University, Evin, Tehran 1983969411, Iran \\ ${ }^{2}$ Center for Correlated Electron Systems, Institute for Basic Science, Seoul 08826, Korea \\ ${ }^{3}$ Department of Physics and Astronomy, Seoul National University, Seoul 08826, Korea \\ ${ }^{4}$ Center for Theoretical Physics (CTP), Seoul National University, Seoul 08826, Korea \\ ${ }^{5}$ Department of Electrical Engineering, Sharif University of Technology, Tehran, Iran
}

\begin{abstract}
We theoretically investigate the magnetoelastic coupling (MEC) and its effect on magnon transport in two-dimensional antiferromagnets with a honeycomb lattice. MEC coefficient along with magnetic exchange parameters and spring constants are computed for monolayers of transitionmetal trichalcogenides with Néel order $\left(\mathrm{MnPS}_{3}\right.$ and $\left.\mathrm{VPS}_{3}\right)$ and zigzag order $\left(\mathrm{CrSiTe}_{3}, \mathrm{NiPS}_{3}\right.$ and $\mathrm{NiPSe}_{3}$ ) by ab initio calculations. Using these parameters, we predict that the spin-Nernst coefficient is significantly enhanced due to magnetoelastic coupling. Our study shows that although Dzyaloshinskii-Moriya interaction can produce spin-Nernst effect in these materials, other mechanisms such as magnon-phonon coupling should be taken into account. We also demonstrate that the magnetic anisotropy is an important factor for control of magnon-phonon hybridization and enhancement of the Berry curvature and thus the spin-Nernst coefficient. Our results pave the way towards gate tunable spin current generation in 2D magnets by SNE via electric field modulation of MEC and anisotropy.
\end{abstract}

Recently, there is a growing interest in antiferromagnets (AFMs) as promising material platforms in spintronics. Because of the intrinsic timescale at $\mathrm{THz}$ range and the absence of the stray field, antiferromagnetic materials specially two dimensional (2D) AFMs [1-3] due to their reduced dimension are excellent candidates for highspeed and compact devices. Several interesting phenomena have been studied on AFMs such as the spin Hall effect [4-7], thermal Hall effect [8-10] and spin Nernst effect (SNE)[11-15]. In case of SNE, an experimental demonstration in $\mathrm{MnPS}_{3}$ was first attributed to the presence of the Dzyaloshinskii-Moriya interaction (DMI) [16]. However, the $\mathrm{DMI}$ in $\mathrm{MnPS}_{3}$ was later found to be too small to be responsible for this effect [17]. In one hand, it is well known that the Berry curvature plays a crucial role on the transport of collective excitations in various systems [18-20]. On the other hand, a magnetoelastic wave (hybridized excitation of magnons and phonons [21]), can carry large Berry curvature in the anticrossing regions between the magnon and phonon bands. These excitations which originate from the magnetoelastic coupling (MEC), can exhibit nontrivial topology even in the systems with trivial topology [22-24]. Therefore, the MEC can be an important mechanism for inducing large SNE in AFMs [46].

MEC has been considered in ferromagnetic materials with a square [25] or honeycomb lattice [26] and in AFMs with a square lattice [27] in both magnon and phonon

\footnotetext{
* Present address: Laboratory of Nanoscale Magnetic Materials and Magnonics (LMGN), Institute of Materials (IMX), School of Engineering (STI), EPFL, 1015 Lausanne, Switzerland.; mohamad.hamdi90@gmail.com

$\dagger$ m-mohseni@sbu.ac.ir

¥ ali_sadeghi@sbu.ac.ir
}

transport properties. Though these studies provide insight on the importance of the MEC in magnon transport, they do not deal with the realistic materials. In this work, we choose transition-metal trichalcogenides (TMTCs) with honeycomb magnetic lattices because several magnetic phases especially AFM orders have been observed in these materials. Moreover, TMTCs are layered compounds with weak interlayer van der Waals interactions and are therefore excellent candidates for $2 \mathrm{D}$ AFMs [28-34].

We introduce a four-state method for calculating the MEC coefficients based on first-principles calculations in the framework of the density functional theory (DFT). Force constants are calculated by finite difference [35] while the values for symmetric exchange interactions up to the third nearest neighbor as well as the second nearest neighbor DMI interactions are taken from Ref. 15 . Using these parameters we obtain the SNE coefficient for Néel $\left(\mathrm{MnPS}_{3}\right.$ and $\left.\mathrm{VPS}_{3}\right)$ and zigzag $\left(\mathrm{CrSiTe}_{3}, \mathrm{NiPS}_{3}\right.$ and $\mathrm{NiPSe}_{3}$ ) ordered TMTCs. Our results show that including the MEC enhances the SNE coefficients by one or two orders of magnitude. Furthermore, our study shows that magnetic anisotropy plays a crucial role in the magnonphonon hybridization and thus SNE. These findings suggest a way for tunability and control of SNE generated spin current via modulation of MEC and anisotropy with e.g. applying a gate voltage.

We perform DFT $+\mathrm{U}$ calculations as implemented in QuANTum ESPRESSO [36] with the Rappe-RabeKaxiras-Joannopoulos ultrasoft (RRKJUS) pseudopotentials, the Perdew-Burke-Ernzerhof (PBE) formalism of the generalized gradient approximation (GGA) [37] and a kinetic energy cutoff of $400 \mathrm{eV}$. The Hubbard potential parameters are assumed as 5, 3.25, 4 and $6.45 \mathrm{eV}$ for $\mathrm{Mn}, \mathrm{V}, \mathrm{Cr}$ and $\mathrm{Ni}$, respectively [32]. A tight convergence threshold of $10^{-7} \mathrm{eV}$ is assumed for the total 


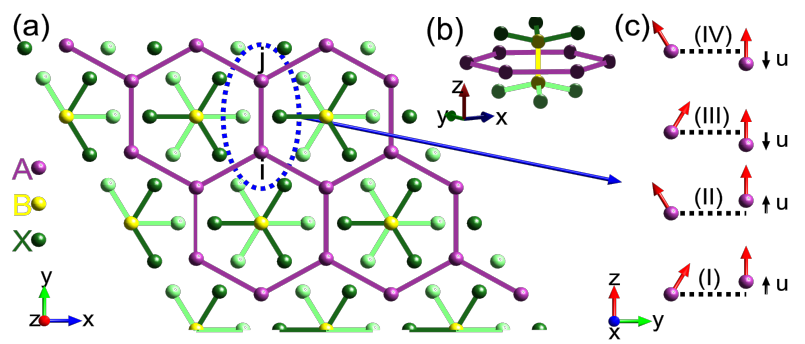

FIG. 1. (a) Two-dimensional view of a $3 \times 3$ supercell representing a TMTC $\left(\mathrm{ABX}_{3}\right)$ material where $\mathrm{A}, \mathrm{B}$ and $\mathrm{X}$ atoms are shown with purple, yellow and green spheres, respectively. (b) Side view of the material depicting $\mathrm{B}_{2} \mathrm{X}_{6}$ ligands located on the center of a hexagon made by A atoms. (c) Schematic representation of the four states used to calculate the MEC coefficient. The big arrows represent the spin of two neighbouring magnetic atoms while the smaller one indicates the out-of-plane displacement of one of them.

energy. A vacuum region larger than $20 \AA$ is used for each slab. Structural optimization are done while fixing the in-plane lattice constants to those reported in Refs. 31 and 32. The magnetic ions A (Mn, V, Cr, Ni) make a honeycomb lattice with $\mathrm{B}_{2} \mathrm{X}_{6}$ ligands on the center of hexagons, as illustrated in Fig. 1 (a-b). We take a $3 \times 3$ supercell to make a separation between the periodic images of the deformed sites as large as $\sim 18 \AA$, and a $k$-point grid of $3 \times 3 \times 1$ for sampling the Brillouin zone.

In the investigated TMTCs with honeycomb magnetic lattice the spins possess Néel or zigzag ordering with out of plane spin orientation. The magnetic atoms can move out of plane and thus MEC comes into the play. Total Hamiltonian reads $H=H_{\mathrm{m}}+H_{\mathrm{ph}}+H_{\mathrm{mp}}$. We write the magnetic Hamiltonian as

$$
\begin{aligned}
H_{\mathrm{m}} & =J_{1} \sum_{\langle i j\rangle} \mathbf{S}_{i} . \mathbf{S}_{j}+J_{2} \sum_{\langle\langle i j\rangle\rangle} \mathbf{S}_{i} \cdot \mathbf{S}_{j}+J_{3} \sum_{\langle\langle\langle i j\rangle\rangle\rangle} \mathbf{S}_{i} . \mathbf{S}_{j} \\
& +D_{2} \sum_{\langle\langle i j\rangle\rangle} \xi_{i j} \hat{\mathbf{z}} .\left(\mathbf{S}_{i} \times \mathbf{S}_{j}\right)+\mathcal{K} \sum_{i} S_{i z}^{2}-\mu \sum_{i} \mathbf{S}_{i} \cdot \mathbf{H}
\end{aligned}
$$

where $J_{1}, J_{2}$ and $J_{3}$ are the Heisenberg exchange interaction parameters between the first, second, and third nearest neighbor spins, respectively. $D_{2}$ is the DMI parameter in the $z$ direction, $\mathbf{S}_{i}$ is the total spin at site $i$, and $\xi_{i j}=\operatorname{sgn} \sum_{\langle i, k\rangle,\langle k, j\rangle} \hat{\mathbf{z}} \cdot\left(\mathbf{r}_{i k} \times \mathbf{r}_{k j}\right)$, where $\mathbf{r}_{i j}=\mathbf{r}_{j}-\mathbf{r}_{j}$ with $\mathbf{r}_{i}$ denoting the position of site $i$. The last two terms in Eq. (1) correspond to the easy-axis anisotropy and Zeeman coupling to external magnetic field, respectively. We apply the Holstein-Primakoff transformation [38] for spin $\mathbf{S}_{i}=S_{i}^{x} \hat{\mathbf{x}}_{i}+S_{i}^{y} \hat{\mathbf{y}}_{i}+S_{i}^{z} \hat{\mathbf{z}}_{i}$ where $S_{i}^{x}=\sqrt{2 S}\left(a_{i}+a_{i}^{\dagger}\right) / 2$, $S_{i}^{y}=\sqrt{2 S}\left(a_{i}-a_{i}^{\dagger}\right) / 2 i$ and $S_{i}^{z}=S-a_{i} a_{i}^{\dagger}$. The local spin coordinates for upward spins coincide the global coordinates while for downward spins $\hat{\mathbf{x}}_{i}=\hat{\mathbf{x}}, \hat{\mathbf{y}}_{i}=-\hat{\mathbf{y}}$ and $\hat{\mathbf{z}}_{i}=-\hat{\mathbf{z}}$.

For each magnetic atom of mass $M$, only its out-ofplane displacements, $u_{i}$, is relevant to the MEC [27]. The corresponding phonon Hamiltonian is therefore given by

$$
H_{\mathrm{ph}}=\frac{1}{2 M} \sum_{i} p_{i}^{2}+\frac{K_{1}^{z}}{2} \sum_{\langle i j\rangle} u_{i j}^{2}
$$

where $p_{i}$ denotes the linear momentum of atom $i, K_{1}^{z}$ is the nearest neighbor transverse spring constant and $u_{i j}=$ $u_{i}-u_{j}$ is correspondingly the distance between the outof-plane positions of two nearest neighbors. Finally, the magnon-phonon coupling part of the Hamiltonian arising from MEC in an AFM honeycomb lattice is described to the linear order by $[25,26,39]$

$$
H_{\mathrm{mp}}=\kappa \sum_{\langle i j\rangle} \lambda_{i}\left(\mathbf{S}_{i} \cdot \hat{\mathbf{r}}_{i j}\right) u_{i j}
$$

where $\kappa$ is the magnetoelastic constant and $\hat{\mathbf{r}}_{i j}=\mathbf{r}_{i j} / r_{i j}$. Note that Eq. (3) is valid only if $\lambda_{i}=S_{i}^{z} / S \approx \pm 1$.

The numerical values of coefficients in Eqs. (1), (2) and (3) are listed in Table I. The values of $J_{1}, J_{2}, J_{3}$ and $D_{2}$ are those previously determined from first principles calculations by a four-state method Ref. $15 . K_{1}^{z}$ is calculated by finite difference method from DFT $+\mathrm{U}$ energies. For calculating $\kappa$, we adopt the following four-state method. In short, all the spins in the supercell are set parallel to the $z$-axis while the spin of atom at a given site $i$ is slightly tilted toward or away from a nearest neighbor atom at site $j$ which is displaced normal to the sheet plane either upward or downward by a tiny value, e.g. $u=0.05 \AA$. The four possible configurations of a pair of atoms highlighted in Fig. 1(a) are:

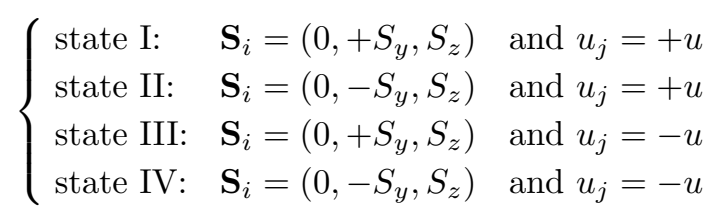

as illustrated schematically in Fig. 1(c). A value of $S_{y}=$ $\pm 0.3 S$ which leads to $\lambda_{i}= \pm 0.954$, fulfills the validity requirements for Eq. (3) as discussed before. It can be easily shown that the MEC coefficient reads

$$
\kappa=\frac{1}{4 S_{y} u}\left(E_{\mathrm{II}}+E_{\mathrm{III}}-E_{\mathrm{I}}-E_{\mathrm{IV}}\right) .
$$

where $E_{\mathrm{I}}, E_{\mathrm{II}}, E_{\mathrm{III}}$ and $E_{\mathrm{IV}}$ are the $\mathrm{DFT}+\mathrm{U}$ energies of the four states defined in Eq. (4).

To obtain the band structure of the excitations the total bosonic BdG Hamiltonian in the Fourier space,

$$
H=\frac{1}{2} \sum_{\mathbf{k}} \phi_{\mathbf{k}}^{\dagger} \mathcal{H}(\mathbf{k}) \phi_{\mathbf{k}}
$$

is diagonalized using the Colpa's method [15, 24, 44] (for 
TABLE I. Magnetic ground state, lattice constant $(\AA)$, magnetic moment per TM atom, exchange (meV) and DMI parameters $(\mu \mathrm{eV})$, Néel temperature $(\mathrm{K})$ from Monte Carlo simulations and experiment, anisotropy coefficient (meV), transverse spring constant $\left(\mathrm{meV} / \AA^{2}\right)$ and MEC coefficient $(\mathrm{meV} / \AA)$ for the five investigated TMTCs. The two columns on the right are calculated in this work. The first eight columns are adopted from Ref. [15]. $\mathcal{K}(\mathrm{meV})$ is set to the available experimental values for bulk $\mathrm{MnPS}_{3}$ [29] and $\mathrm{NiPS}_{3}$ [40]. For $\mathrm{NiPSe}_{3}$ and $\mathrm{VPS}_{3}$ no experimental $\mathcal{K}$ is available while the experimental values for bulk $\mathrm{CrSiTe}_{3}$ [41] makes our 2D calculation numerically unstable (We note that the GS 2D CrSiTe 3 is zigzag [31] while it is ferromagnetic in bulk [41]). Therefore, for $\mathrm{NiPSe}_{3}$ we use the same $\mathcal{K}$ value as $\mathrm{NiPS}_{3}$, and for $\mathrm{VPS}_{3}$ and $\mathrm{CrSiTe}_{3}$ we took the smallest values that lead to stable numerical solutions.

\begin{tabular}{|c|c|c|c|c|c|c|c|c|c|c|c|c|}
\hline \multicolumn{4}{|c|}{$\overline{\text { Structure }}$} & \multicolumn{4}{|c|}{ Exchange and DMI } & \multicolumn{2}{|c|}{ Néel Temp. } & \multirow[b]{2}{*}{$\mathcal{K}$} & \multirow[b]{2}{*}{$K_{1}^{z}$} & \multirow[b]{2}{*}{$|\kappa|$} \\
\hline Material & GS & $a$ & $S / \mu_{B}$ & $J_{1}$ & $J_{2}$ & $J_{3}$ & $\overline{D_{2 z}}$ & $\mathrm{MC}$ & Exp & & & \\
\hline$\overline{\mathrm{MnPS}}_{3}$ & Néel & 5.88 & 4.56 & 0.527 & 0.024 & 0.150 & 0.39 & 150 & $78[29]$ & $\overline{-0.002}$ & $\overline{479.9}$ & 0.0292 \\
\hline $\mathrm{VPS}_{3}$ & Néel & 5.85 & 2.82 & 7.387 & 0.068 & 0.223 & 7.23 & 530 & - & -0.014 & 106.7 & 0.6858 \\
\hline $\mathrm{CrSiTe}_{3}$ & zigzag & 6.84 & 3.76 & -0.990 & 0.009 & 0.389 & 39.00 & 48 & $80[42]$ & -0.023 & 118.9 & 0.9320 \\
\hline $\mathrm{NiPS}_{3}$ & zigzag & 5.82 & 1.58 & -1.039 & -0.163 & 3.882 & 4.54 & 105 & $150[43]$ & -0.190 & 243.6 & 0.2949 \\
\hline $\mathrm{NiPSe}_{3}$ & zigzag & 6.14 & 1.56 & -1.131 & -0.069 & 3.975 & 43.90 & 110 & - & -0.190 & 173.8 & 1.9729 \\
\hline
\end{tabular}
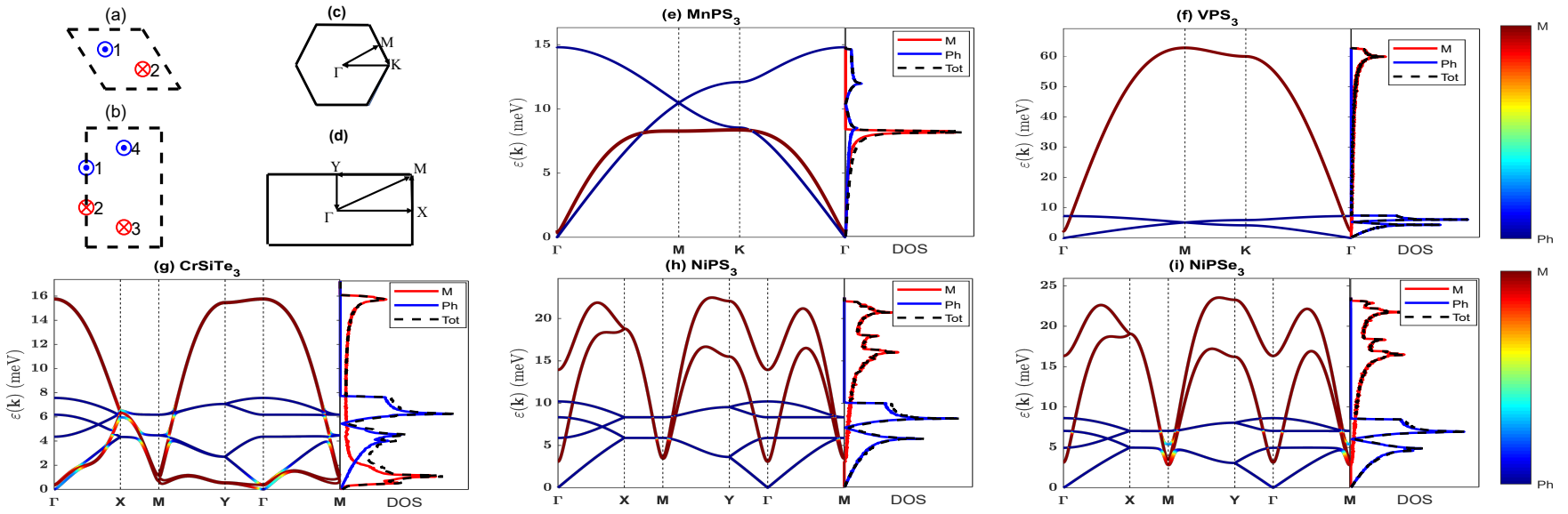

FIG. 2. Magnetic unit cell for (a) the Néel phase and (b) zigzag phase. Symmetry points for (c) the Néel phase and (d) zigzag phase. The band structure and density of states (DOS) of (e) $\mathrm{MnPS}_{3}$, (f) $\mathrm{VPS}_{3}$, (g) $\mathrm{CrSiTe}_{3}$, (h) $\mathrm{NiPS}_{3}$ and (i) NiPSe 3 . The spin character $\left|\left\langle S^{z}\right\rangle\right|$ of the modes is indicated with colors.

details, see SM [45])

$$
\Psi^{\dagger}(\mathbf{k}) \mathcal{H}(\mathbf{k}) \Psi(\mathbf{k})=E(\mathbf{k})
$$

The elements of $2 N \times 2 N$ diagonal matrix $E(\mathbf{k})$ are the eigenenergies while the associated eigenstates are the columns of $\Psi(\mathbf{k})$ and satisfy $\hat{\varphi} \mathcal{H}(\mathbf{k})|n, \mathbf{k}\rangle=E_{n n}(\mathbf{k})|n, \mathbf{k}\rangle$ and $\langle n, \mathbf{k}|\hat{\varphi}| m, \mathbf{k}\rangle=\hat{\varphi}_{n m}$. Note that $\Psi(\mathbf{k})$ is a paraunitary matrix i.e. it satisfies $\Psi^{\dagger}(\mathbf{k}) \hat{\varphi}=\hat{\varphi} \Psi^{-1}(\mathbf{k})$, where $\hat{\varphi}=\operatorname{diag}(1, \cdots, 1,-1, \cdots,-1)$. For the Néel and zigzag orders, shown in Figs. 2(a-b), $N=4$ and 8, respectively.

Since the spin is not conserved in the presence of MEC, the semiclassical approach is not valid and linear response theory should be applied [46]. For this purpose, a generalized Berry curvature should be defined for operator $\theta=\frac{1}{4}\left(S^{z} \hat{\varphi} \mathbf{v}+\mathbf{v} \hat{\varphi} S^{z}\right)$ where $\mathbf{v}=\hbar^{-1} \partial_{\mathbf{k}} H$ is the velocity operator and $S^{z}=-\sum_{i}^{N / 2} \lambda_{i} a_{i}^{\dagger} a_{i}$ is the magnon spin operator. The generalized Berry curvature of the $n$th band is given by [47]

$$
\left(\Omega_{n}^{\theta}(\mathbf{k})\right)_{x y}=\sum_{m \neq n} \frac{2 i\left\langle n\left|\theta_{x}\right| m\right\rangle\left\langle m\left|v_{y}\right| n\right\rangle(\hat{\varphi})_{n n}(\hat{\varphi})_{m m}}{\left((\hat{\varphi} E)_{n n}-(\hat{\varphi} E)_{m m}\right)^{2}}
$$

where the index $\mathbf{k}$ is dropped for simplicity (the conventional Berry curvature is presented in the SM [45].) The SNE coefficient is defined as $\alpha_{x y}^{s} \equiv \mathbf{j}_{S N E} /(\hat{z} \times \nabla T)$ [11], where $\nabla T$ is an in-plane temperature gradient and the magnon spin current, $\mathbf{j}_{S N E}$ is calcauted based on the linear response theory $[46,47]$ as

$$
\alpha_{x y}^{s}=2 k_{B} \sum_{n=1}^{N} \int d \mathbf{k}\left(\Omega_{n}^{\theta}(\mathbf{k})\right)_{x y} c_{1}\left(\frac{E_{n n}}{k_{B} T}\right)
$$

where $c_{1}(x)=[1+\rho(x)] \log [1+\rho(x)]-\rho(x) \log \rho(x)$ and $\rho(x)=1 /\left(e^{x}-1\right)$. Note that only the particle bands (the first $N$ bands) contribute to the summation.

In an experimental report a bulk crystal of $\mathrm{MnPS}_{3}$ was used whose edges are cut at an inclination angle $\eta=45^{\circ}$ and $\mathrm{Pt}$ stripes were used as electrodes [16]. To get results 
comparable with such SNE measurement in TMTCs, we adopt the SNE signal introduced in Ref. 15

$$
\mathcal{S}_{S N E}=\frac{V_{S N E}}{\Delta T}=\frac{e}{\hbar d} \rho \theta_{S H} \alpha_{x y}^{s} \cos \eta .
$$

where $V_{S N E}$ is voltage due to inverse spin Hall effect, $\Delta T$ is the temperature difference across the length of $\mathrm{Pt}$ stripes, $d$ is the inter-layer distance in the TMTC, and $\rho=10^{-6} \Omega / \mathrm{m}$ and $\theta_{S H}=0.15$ are the electrical resistivity and the spin Hall angle of $\mathrm{Pt}$, respectively.

All the parameters for constructing the Hamiltonian, including the calculated MEC coefficients $\kappa$ and transverse spring constant $K_{1}^{z}$, are reported in Table I. The resulting band structure along the high symmetry directions shown in Figs. 2(c) and (d) as well as the density of states (DOS) of the quasiparticle bands are presented in Figs. 2(e-f) and (g-i) for the Néel and zigzag ordered materials, respectively. From the absolute value of the z-component of spin, $\left|\left\langle S^{z}\right\rangle\right|$ [45], encoded by the color bar in the band structure plots, one clearly identifies two degenerate magnon bands (red) and two phonon bands (blue) in the Néel phase. On the other hand, four magnon bands (two doubly-degenerate bands) and four phonon bands are identified for the zigzag phase.

As can be seen in Fig. 2, MEC leads to hybridization of magnon and phonon bands which manifests itself as the anticrossing regions (some of anticrossing regions are shown in Fig. S6). Therefore, the generalized Berry curvature becomes very large in these anticrossing regions for both Néel and zigzag ordered materials, as illustrated in Figs. S3 and S4 of the SM [45]. Consequently, the SNE coefficient is largely increased as seen in Fig. 3. By including the MEC in the calculations, the maximum SNE coefficient within the shown temperature range is enhanced by a factor of $\sim 23,12,13,620$ and 99 for $\mathrm{MnPS}_{3}, \mathrm{VPS}_{3}, \mathrm{CrSiTe}_{3}, \mathrm{NiPS}_{3}$ and $\mathrm{NiPSe}_{3}$, respectively.

A log-log plot of the absolute value of SNE coefficient, $\left|\alpha_{x y}^{s}\right|$, as a function of the anisotropy $\mathcal{K}$ at $T=50 \mathrm{~K}$ is also depicted in the insets of Fig. 3 (Note that there is a sign change at $\mathcal{K}=-0.004 \mathrm{meV}$ for $\mathrm{MnPS}_{3}$ ). It is clearly seen that the SNE coefficient is fairly sensitive to changes in the anisotropy. This is due to the effect of anisotrpy in displacing bands and thus anticrossing regions which in turn changes the generalized Berry curvature and SNE.

There are also other factors that affect SNE coefficient. For $\mathrm{MnPS}_{3}$ and $\mathrm{VPS}_{3}$, there are anticrossing region at higher energies (around $8 \mathrm{meV}$ ) leading to different behavior at higher temperatures (around $90 \mathrm{~K}$ ). The situation is more complicated for zigzag ordered materials due to magnon-phonon hybridization in several regions. Specially, for $\mathrm{CrSiTe}_{3}$ we can see large generalized Berry curvature almost everywhere in the Brillouin zone (Fig. S4) which leads to very large SNE coefficient compared to other materials. For $\mathrm{NiPS}_{3}$ and $\mathrm{NiPSe}_{3}$, we see anticrossing regions around the $\mathrm{M}$ and $\Gamma$ points (See Figs. 2(h) and (i)). However, relatively small generalized Berry curvature is induced near the $\Gamma$ point (see Fig. S4). We thus consider only the anticrossing regions around the $\mathrm{M}$ point. For both materials the hybridization is stronger at the anticrossing with lower energies. However, since the MEC coefficient $\kappa$ of $\mathrm{NiPSe}_{3}$ is very large, the magnon bands which carry +1 and -1 spins and the phonon bands strongly mix at this region (around the $\mathrm{M}$ point). This results in magnon bands with almost no spin, i.e. the spin sectors with opposite spins cancel each other (shown in Figs. S5 (n) and (o) where the lower bands around the $\mathrm{M}$ point have considerable magnon contents but almost no spin), so they have less contribution to the SNE coefficient. On the other hand, $\kappa$ of $\mathrm{NiPS}_{3}$ is much smaller, thus there are still bands that can carry spin at the anticrossings around the $\mathrm{M}$ point with lower energies. So the lower bands mainly contribute to the SNE coefficient (see Fig. S4), and their contribution is negative leading to negative SNE coefficient. In contrast, the anticrossings around the $\mathrm{M}$ point with higher energies (higher bands at it can be seen in Fig. S4) contributes mainly to the SNE coefficient of $\mathrm{NiPS}_{3}$ and their contribution is positive.

We also note that the individual contribution of each band to SNE coefficient can be orders of magnitude larger than the final SNE (see Fig. S7). However, the sum of positive bands is almost equal to negative bands but opposite in sign (see the inset of Fig. S7). So there is very small difference between the absolute values of positive and negative bands which leads to very small SNE coefficient compared to the contribution of each band yet much larger than SNE coefficient in the absence of MEC. In contrast, when $\kappa=0$ the contribution of each band is comparable with final SNE coefficient.

In summary, this study reveals that MEC induces large generalized Berry curvatures in anticrossing regions which in turn translates into large SNE in TMTC materials. We also found that the SNE is sensitive to anisotropy and paves the way for designing tunable spintronic devices. Among the TMTC materials studied in this work, $\mathrm{NiPSe}_{3}$ has the largest MEC, but the largest SNE is observed in $\mathrm{CrSiTe}_{3}$. Despite the focus of previous studies, presence of DMI cannot explain large SNE observed in $\mathrm{MnPS}_{3}$ [16]. Other magnon-phonon coupling mechanisms should be also considered in the future works to better understand the topological properties of TMTC antiferromagnets.

Acknowledgment. This project is funded by Iran Science Elites Federation (ISEF). The support and computatioanl resources from the Center for High Performance Computing (SARMAD) at SBU is gratefully acknowledged. S. P. was supported by the Institute for Basic Science in Korea (Grant No. IBS-R009-D1), Samsung Science and Technology Foundation under Project Number SSTF-BA2002-06, the National Research Foundation of Korea (NRF) grant funded by the Korea government (MSIT) (No.2021R1A2C4002773, and No. NRF2021R1A5A1032996). 


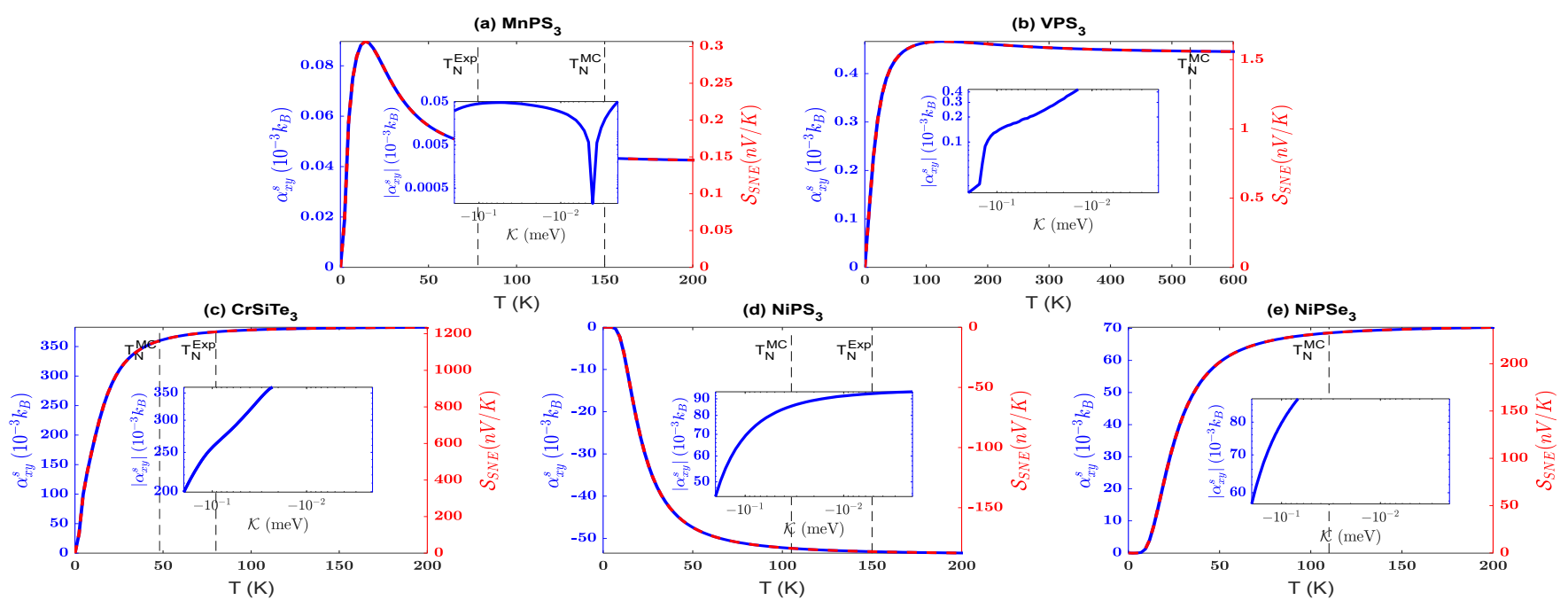

FIG. 3. The SNE coefficient (blue dashed lines) and SNE signal (red dashed lines) versus temperature, top for Néel phase and bottom for zigzag phase. The experimental and Monte Carlo Néel temperatures are also marked with vertical black dashed lines. Insets: The SNE coefficient in terms of easy-axis anisotropy $\mathcal{K}$ plotted in logrithmic scale.

[1] Tomas Jungwirth, X Marti, P Wadley, and J Wunderlich, "Antiferromagnetic spintronics," Nature nanotechnology 11, 231-241 (2016).

[2] Matthias B Jungfleisch, Wei Zhang, and Axel Hoffmann, "Perspectives of antiferromagnetic spintronics," Physics Letters A 382, 865-871 (2018).

[3] V Baltz, Aurelien Manchon, M Tsoi, T Moriyama, T Ono, and Y Tserkovnyak, "Antiferromagnetic spintronics," Reviews of Modern Physics 90, 015005 (2018).

[4] Yongxi Ou, Shengjie Shi, DC Ralph, and RA Buhrman, "Strong spin hall effect in the antiferromagnet ptmn," Physical Review B 93, 220405 (2016).

[5] Wei Zhang, Matthias B Jungfleisch, Wanjun Jiang, John E Pearson, Axel Hoffmann, Frank Freimuth, and Yuriy Mokrousov, "Spin hall effects in metallic antiferromagnets," Physical review letters 113, 196602 (2014).

[6] JBS Mendes, RO Cunha, O Alves Santos, PRT Ribeiro, FLA Machado, RL Rodríguez-Suárez, A Azevedo, and SM Rezende, "Large inverse spin hall effect in the antiferromagnetic metal ir 20 mn 80," Physical Review B 89, 140406 (2014).

[7] Motoi Kimata, Hua Chen, Kouta Kondou, Satoshi Sugimoto, Prasanta K Muduli, Muhammad Ikhlas, Yasutomo Omori, Takahiro Tomita, Allan H MacDonald, Satoru Nakatsuji, et al., "Magnetic and magnetic inverse spin hall effects in a non-collinear antiferromagnet," Nature 565, 627-630 (2019).

[8] Masataka Kawano and Chisa Hotta, "Thermal hall effect and topological edge states in a square-lattice antiferromagnet," Physical Review B 99, 054422 (2019).

[9] Alexander Mook, Jürgen Henk, and Ingrid Mertig, "Thermal hall effect in noncollinear coplanar insulating antiferromagnets," Physical Review B 99, 014427 (2019).

[10] Kyung-Su Kim, Ki Hoon Lee, Suk Bum Chung, and JeGeun Park, "Magnon topology and thermal hall effect in trimerized triangular lattice antiferromagnet," Physical
Review B 100, 064412 (2019).

[11] Ran Cheng, Satoshi Okamoto, and Di Xiao, "Spin nernst effect of magnons in collinear antiferromagnets," Physical review letters 117, 217202 (2016).

[12] Vladimir A Zyuzin and Alexey A Kovalev, "Magnon spin nernst effect in antiferromagnets," Physical review letters 117, 217203 (2016).

[13] Guang-Yu Guo and Tzu-Cheng Wang, "Large anomalous nernst and spin nernst effects in the noncollinear antiferromagnets mn $3 \mathrm{x}(\mathrm{x}=\mathrm{sn}$, ge, ga $), "$ Physical Review B 96, 224415 (2017).

[14] Yinhan Zhang, Satoshi Okamoto, and Di Xiao, "Spinnernst effect in the paramagnetic regime of an antiferromagnetic insulator," Physical Review B 98, 035424 (2018).

[15] N Bazazzadeh, M Hamdi, F Haddadi, A Khavasi, A Sadeghi, and SM Mohseni, "Symmetry enhanced spinnernst effect in honeycomb antiferromagnetic transition metal trichalcogenide monolayers," Physical Review B 103, 014425 (2021).

[16] Y Shiomi, R Takashima, and E Saitoh, "Experimental evidence consistent with a magnon nernst effect in the antiferromagnetic insulator mnps 3," Physical Review B 96, 134425 (2017).

[17] AR Wildes, Satoshi Okamoto, and Di Xiao, "Search for nonreciprocal magnons in mnps 3," Physical Review B 103, 024424 (2021).

[18] Naoto Nagaosa, Jairo Sinova, Shigeki Onoda, Allan H MacDonald, and Nai Phuan Ong, "Anomalous hall effect," Reviews of modern physics 82, 1539 (2010).

[19] Di Xiao, Ming-Che Chang, and Qian Niu, "Berry phase effects on electronic properties," Reviews of modern physics 82, 1959 (2010).

[20] Taishi Chen, Takahiro Tomita, Susumu Minami, Mingxuan Fu, Takashi Koretsune, Motoharu Kitatani, Ikhlas Muhammad, Daisuke Nishio-Hamane, Rieko Ishii, Fu- 
miyuki Ishii, et al., "Anomalous transport due to weyl fermions in the chiral antiferromagnets mn $3 \mathrm{x}, \mathrm{x}=\mathrm{sn}$, ge," Nature communications 12, 1-14 (2021).

[21] Naoki Ogawa, Wataru Koshibae, Aron Jonathan Beekman, Naoto Nagaosa, Masashi Kubota, Masashi Kawasaki, and Yoshinori Tokura, "Photodrive of magnetic bubbles via magnetoelastic waves," Proceedings of the National Academy of Sciences 112, 8977-8981 (2015).

[22] Ryuji Takahashi and Naoto Nagaosa, "Berry curvature in magnon-phonon hybrid systems," Physical review letters 117, 217205 (2016).

[23] Xiaoou Zhang, Yinhan Zhang, Satoshi Okamoto, and Di Xiao, "Thermal hall effect induced by magnon-phonon interactions," Physical review letters 123, 167202 (2019).

[24] Sungjoon Park and Bohm-Jung Yang, "Topological magnetoelastic excitations in noncollinear antiferromagnets," Physical Review B 99, 174435 (2019).

[25] Gyungchoon Go, Se Kwon Kim, and Kyung-Jin Lee, "Topological magnon-phonon hybrid excitations in twodimensional ferromagnets with tunable chern numbers," Physical review letters 123, 237207 (2019).

[26] Even Thingstad, Akashdeep Kamra, Arne Brataas, and Asle Sudbø, "Chiral phonon transport induced by topological magnons," Physical review letters 122, 107201 (2019).

[27] Shu Zhang, Gyungchoon Go, Kyung-Jin Lee, and Se Kwon Kim, "Su (3) topology of magnon-phonon hybridization in $2 \mathrm{~d}$ antiferromagnets," Physical review letters 124, 147204 (2020).

[28] A Wiedenmann, J Rossat-Mignod, A Louisy, R Brec, and J Rouxel, "Neutron diffraction study of the layered compounds mnpse3 and fepse3," Solid State Communications 40, 1067-1072 (1981).

[29] AR Wildes, B Roessli, B Lebech, and KW Godfrey, "Spin waves and the critical behaviour of the magnetization in," Journal of Physics: Condensed Matter 10, 6417 (1998).

[30] B Siberchicot, S Jobic, V Carteaux, P Gressier, and G Ouvrard, "Band structure calculations of ferromagnetic chromium tellurides crsite 3 and crgete3," The Journal of Physical Chemistry 100, 5863-5867 (1996).

[31] Nikhil Sivadas, Matthew W Daniels, Robert H Swendsen, Satoshi Okamoto, and Di Xiao, "Magnetic ground state of semiconducting transition-metal trichalcogenide monolayers," Physical Review B 91, 235425 (2015).

[32] Bheema Lingam Chittari, Youngju Park, Dongkyu Lee, Moonsup Han, Allan H MacDonald, Euyheon Hwang, and Jeil Jung, "Electronic and magnetic properties of single-layer m p x 3 metal phosphorous trichalcogenides," Physical Review B 94, 184428 (2016).

[33] PA Joy and S Vasudevan, "Magnetism in the layered transition-metal thiophosphates $\mathrm{m}$ ps $3(\mathrm{~m}=\mathrm{mn}$, fe, and ni)," Physical Review B 46, 5425 (1992).

[34] Y Takano, N Arai, A Arai, Y Takahashi, K Takase, and K Sekizawa, "Magnetic properties and specific heat of

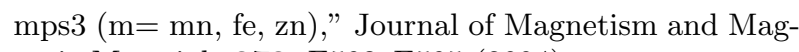
netic Materials 272, E593-E595 (2004).

[35] Johannes Sarnthein, Alfredo Pasquarello, and Roberto Car, "Origin of the high-frequency doublet in the vibrational spectrum of vitreous sio2," Science 275, 1925-1927 (1997).

[36] Paolo Giannozzi, Stefano Baroni, Nicola Bonini, Matteo Calandra, Roberto Car, Carlo Cavazzoni, Davide Ceresoli, Guido L Chiarotti, Matteo Cococcioni, Ismaila Dabo, et al., "Quantum espresso: a modular and opensource software project for quantum simulations of materials," Journal of physics: Condensed matter 21, 395502 (2009).

[37] John P Perdew, Kieron Burke, and Matthias Ernzerhof, "Generalized gradient approximation made simple," Physical review letters 77, 3865 (1996).

[38] Wolfgang Nolting and Anupuru Ramakanth, Quantum theory of magnetism (Springer Science \& Business Media, 2009).

[39] Ch Kittel, "Interaction of spin waves and ultrasonic waves in ferromagnetic crystals," Physical Review 110, 836 (1958).

[40] D Lançon, RA Ewings, $T$ Guidi, F Formisano, and AR Wildes, "Magnetic exchange parameters and anisotropy of the quasi-two-dimensional antiferromagnet nips 3," Physical Review B 98, 134414 (2018).

[41] Travis Williams, Adam Aczel, Brenden Ortiz, Paul Sarte, and Stephen Wilson, "Magnetic correlations in the quasi2d semiconducting ferromagnet crgete 3," Bulletin of the American Physical Society 65 (2020).

[42] Ming-Wei Lin, Houlong L Zhuang, Jiaqiang Yan, Thomas Zac Ward, Alexander A Puretzky, Christopher M Rouleau, Zheng Gai, Liangbo Liang, Vincent Meunier, Bobby G Sumpter, et al., "Ultrathin nanosheets of crsite 3: a semiconducting two-dimensional ferromagnetic material," Journal of Materials Chemistry C 4, 315322 (2016).

[43] Kangwon Kim, Soo Yeon Lim, Jae-Ung Lee, Sungmin Lee, Tae Yun Kim, Kisoo Park, Gun Sang Jeon, CheolHwan Park, Je-Geun Park, and Hyeonsik Cheong, "Suppression of magnetic ordering in xxz-type antiferromagnetic monolayer nips 3," Nature communications 10, 1-9 (2019).

[44] JHP Colpa, "Diagonalization of the quadratic boson hamiltonian," Physica A: Statistical Mechanics and its Applications 93, 327-353 (1978).

[45] See Supplemental Material.

[46] Sungjoon Park, Naoto Nagaosa, and Bohm-Jung Yang, "Thermal hall effect, spin nernst effect, and spin density induced by a thermal gradient in collinear ferrimagnets from magnon-phonon interaction," Nano letters $\mathbf{2 0}$, 2741-2746 (2020).

[47] Bo Li, Shane Sandhoefner, and Alexey A Kovalev, "Intrinsic spin nernst effect of magnons in a noncollinear antiferromagnet," Physical Review Research 2, 013079 (2020). 